\title{
Interaction of porcine gastric mucin with various polycations and its influence on the boundary lubrication properties
}

Nikogeorgos, Nikolaos; Patil, Navinkumar J.; Zappone, Bruno; Lee, Seunghwan

Published in:

Polymer

Link to article, DOI:

10.1016/j.polymer.2016.08.030

Publication date:

2016

Document Version

Peer reviewed version

Link back to DTU Orbit

Citation (APA):

Nikogeorgos, N., Patil, N. J., Zappone, B., \& Lee, S. (2016). Interaction of porcine gastric mucin with various polycations and its influence on the boundary lubrication properties. Polymer, 100, 158-168.

https://doi.org/10.1016/j.polymer.2016.08.030

\section{General rights}

Copyright and moral rights for the publications made accessible in the public portal are retained by the authors and/or other copyright owners and it is a condition of accessing publications that users recognise and abide by the legal requirements associated with these rights.

- Users may download and print one copy of any publication from the public portal for the purpose of private study or research.

- You may not further distribute the material or use it for any profit-making activity or commercial gain

- You may freely distribute the URL identifying the publication in the public portal 


\title{
Interaction of Porcine Gastric Mucin with Various Polycations and Its Influence on the Boundary Lubrication Properties
}

\author{
Nikolaos Nikogeorgos, ${ }^{1}$ Navinkumar J. Patil,${ }^{2}$ Bruno Zappone, ${ }^{3}$ and Seunghwan Lee ${ }^{1, *}$ \\ ${ }^{1}$ Department of Mechanical Engineering, Technical University of Denmark, DK-2800 Kgs. Lyngby, \\ Denmark
}

${ }^{2}$ Dipartimento di Fisica, Università della Calabria, Cubo 33/B, 87036 Rende (CS), Italy

${ }^{3}$ Consiglio Nazionale delle Ricerche - Istituto di Nanotecnologia (CNR-Nanotec), SS Rende LICRYL, c/o Dipartimento di Fisica, Università, della Calabria, Cubo 33/B, Rende (CS) 87036 Italy

*Corresponding author: seele@mek.dtu.dk, +45 45252193

\begin{abstract}
The interaction of porcine gastric mucin (PGM) with polycations possessing amine functional groups, including polyallylamine hydrochloride (PAH), poly-l-lysine (PLL) and polyethyleneimine (PEI), was examined from a tribological standpoint at a self-mated polydimethylsiloxane (PDMS) interface. In all cases, PGM interacted with the cationic polymers, leading to a smaller size of the aggregates compared to PGM, surface charge compensation, and distortion of the PGM protein conformation. But, a synergistic lubrication effect was clearly observed only from the $1 / 1$ (w/w) mixture of PGM with branched PEI (B-PEI) where the friction coefficient was reduced by two
\end{abstract}


orders of magnitude compared to neat PGM or B-PEI in the speed range of $0.25-100 \mathrm{~mm} / \mathrm{s}$. This was attributed to the smallest hydrodynamic size and "sphere-like" conformation of B-PEI due to high degree of branching, which favors tenacious complexation with PGM, fast surface adsorption, and continuous reformation of the lubricating layer under cyclic tribostress in pin-on-disc tribometry.

Key words: Mucoadhesion, Biolubrication, Mucins, PAH, PEI, PLL

\section{Introduction}

Mucus is a biological hydrogel that coats the respiratory, gastrointestinal, and reproductive tracts. One of its main roles is to protect and lubricate the underlying epithelial tissue surfaces [1]. The main macromolecular components of mucus are mucins, which are responsible for the gel formation and physicochemical properties of mucus. Mucins are composed of a linear protein backbone chain, where the central region is heavily glycosylated whereas $\mathrm{C}$ - and $\mathrm{N}$-termini are mostly unglycosylated. Monomeric mucin molecules are often simplified as a triblock copolymer with alternating hydrophobic-hydrophilic-hydrophobic domains. Due to the distinct amphiphilicity, mucins can readily form hydrophilic layers on hydrophobic surfaces in aqueous solution and have received considerable interest from a biotribological point of view [2-10].

One of the most extensively studied subjects with mucus and mucins is their interaction with hydrophilic polymers of both synthetic and natural origins showing strong interaction, i.e. "mucoadhesion" [11-21]. The focus of those studies is mainly to characterize the interaction mechanism and strength, as motivated by the potential use of mucoadhesive polymers in drug delivery applications. Some examples of polymers that have been frequently examined are chitosan 
[11,12], poly(acrylic acid) (PAA) [13-15], poly(ethylene glycol) [16,17], alginate [18,19], and pectin $[20,21]$. The lubricating properties of mucins [7-10] can be greatly influenced by the interaction with such polymers. For example, we have recently reported that the interaction of PGM with chitosan can lead to an adsorbed layer of greatly improved lubricating and wear resistance properties compared to neat PGM or chitosan at a compliant hydrophobic interface due to electrostatic attraction, compaction of PGM molecules, and strong cohesion of the mucin-polymer aggregates [22]. However, the interaction of PGM with chitosan was studied only at acidic environment $(\mathrm{pH} 3)$ and very low polymer concentration $(0.1 \mathrm{mg} / \mathrm{mL})$ because of the poor solubility of chitosan (chitosan is nearly insoluble in neutral aqueous solution). As electrostatic attraction plays a significant role in the formation of aggregates between PGM and chitosan [22], it is reasonable to assume that similar "synergetic lubricating effects" may occur even in neutral $\mathrm{pH}$ if polycations that are dissolvable in neutral $\mathrm{pH}$ aqueous solutions are employed instead.

In the present study, we have considered a number of polycations displaying high solubility in neutral $\mathrm{pH}$ aqueous conditions, namely polyallylamine hydrochloride (PAH), poly-l-lysine (PLL), and polyethyleneimine (PEI) (both linear and branched), and investigated their interaction with PGM with a focus on the influence on the lubricating properties of PGM-polymer mixtures. While all three types of polycations are expected to show electrostatic attraction with polyanionic PGM at neutral $\mathrm{pH}$, the interaction can be different due to different molecular structures in the amine-based cationic moieties (Scheme 1). PAH has one primary amine per monomer whereas linear PEI (LPEI) has secondary amines only and branched PEI (B-PEI) has a mixture of primary, secondary, and tertiary amines, respectively. Furthermore, PLL has also exclusively primary amines as endmoieties of side alkyl chains, but has secondary amines in the polypeptide backbone. Thus, different $\mathrm{pKa}$ values and degree of ionization of primary, secondary, and tertiary amine groups in these polycations in neutral $\mathrm{pH}$ conditions may result in different strength of electrostatic attraction with 
PGM. Moreover, varying distribution of primary, secondary, and tertiary amine groups as well as hydrophobic moieties in these polycations can have an influence on non-electrostatic interactions with PGM, such as hydrogen bonding or hydrophobic interaction. Lastly, many other factors than the strength of interaction between PGM and the polycations may also influence the lubricating capabilities of their mixtures, such as molecular weight (e.g. PAH and PLL in this study) and polymer architecture (e.g. linear and branched configuration for PEI in this study). Given an array of polycations with varying molecular size/structure and chemical functional groups, this study is expected to provide an insight on the parameters that facilitate the synergetic lubricating effect with PGM.

a)

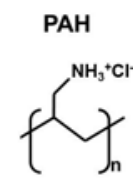

c)

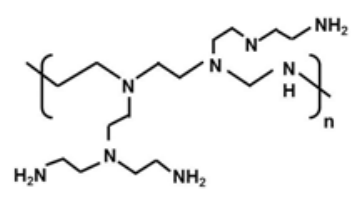

b)
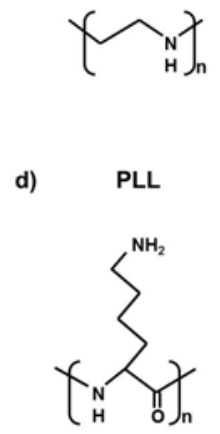

Scheme 1. Molecular structure of the monomeric units of: a) PAH, b) L-PEI, c) B-PEI and d) PLL.

\section{Materials and Methods}

\subsection{Mucins and chemicals}

PGM (Type III: partially purified, bound sialic acid $0.5-1.5 \%$ ), PAH, PEI and PLL were purchased from Sigma-Aldrich (Denmark Aps, Brøndby, Denmark). Two types of PAH with low Mw (15 kDa, product code: 1001791626) and high Mw (900 kDa, product code: 1001758424) were 
employed and denoted as "PAH15" and "PAH900", respectively. Two different kinds of PEI were used, branched (product code: 101535547) and linear (product code: 1001990418), both with $\mathrm{Mn} \approx$ $10 \mathrm{kDa}$, and denoted as "B-PEI10" and "L-PEI10", respectively. The Mw of PLL was either $23 \mathrm{kDa}$ (product code: 100179162) or $230 \mathrm{kDa}$ (product code: 1001791367), and thus they were denoted as "PLL23" and "PLL230", respectively. All chemicals were laboratory grade and purchased also from Sigma Aldrich (Brøndby, Denmark). PGM and the polymer solutions were prepared at a

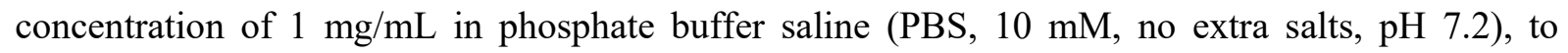
provide a high bulk macromolecule concentration necessary for effective lubrication [10,22]. It should be noted that PAH solutions in PBS displayed turbidity at this concentration (see Figure S1

in Supplementary Data). All PGM:polymer mixtures were prepared from the $1 \mathrm{mg} / \mathrm{mL}$ solutions of PGM and the polymers so that the total polymer concentration [PGM + polymer] in the final mixture remained at $1 \mathrm{mg} / \mathrm{mL}$. Unless otherwise stated, all experiments were started within $1 \mathrm{~h}$ after preparation of the mixture. The $\mathrm{pH}$ of all solutions was maintained at 7.2 using $\mathrm{HCl}$ or $\mathrm{NaOH}$ solution to compensate for the effect of the amine-based polycations.

\subsection{Dialysis of $P G M$}

$500 \mathrm{mg}$ of PGM was dissolved at the concentration of $10 \mathrm{mg} / \mathrm{mL}$ in an aqueous solvent of $10 \mathrm{mM}$ Na-phosphate with pH adjusted at 7.4. The PGM solution was dialyzed in CE BioTech tubing with $100 \mathrm{kDa}$ MWCO (Spectrum Labs) against MilliQ water for $20 \mathrm{~h}$ with solvent exchange after the first 2 h. Sample was then freeze-dried in Christ Alpha 1-2 LD Freeze Dryer (SciQuip) for three days and stored at $-20^{\circ} \mathrm{C}$ until use. 


\subsection{Pin-on-disc tribometry and tribopair}

Tribological investigations were carried out with a pin-on-disc tribometer (CSM, Peseux, Switzerland). A loaded pin was slid in contact with a rotating disc at controlled rotation speeds. The pin was slid over a fixed track (radius: $3 \mathrm{~mm}$ ) under a load $L=1 \mathrm{~N}$ while varying the speed of rotation from 100 to $0.25 \mathrm{~mm} / \mathrm{s}$. The Hertzian contact pressure was estimated to be $0.3 \mathrm{MPa}$ based on the elasticity modulus of $2 \mathrm{MPa}$ and Poisson's ratio of 0.5 for PDMS [10]. The friction force $f$ was determined by a strain gauge. The coefficient of friction was calculated as $\mu=f / L$.

PDMS pins and discs were prepared as follows: the base fluid and crosslinker of Sylgard 184 elastomer kit (Dow Corning, Midland, MI) were mixed at a ratio of 10:1. A gentle vacuum was then applied to remove air trapped in the mixture. Discs were cast in a machined aluminum plate mold with flat wells designed to the dimensions (30 mm diameter $\times 5 \mathrm{~mm}$ thickness) of the disc holder. For the discs, only the sides exposed to air during curing were used for tribological measurements. The pins were molded in a 96 microwell plate (NUNCLON Delta Surface, Roskilde, Denmark) in the same way described for the disc above. The PDMS mixtures were then cured at $70{ }^{\circ} \mathrm{C}$ overnight.

\subsection{Dynamic Light Scattering (DLS)}

The hydrodynamic radii for PGM, PAH15, PAH900, L-PEI10, B-PEI10, PLL23, PLL230 solutions and their mixtures were determined with a Brookhaven ZetaPALS instrument (Brookhaven Instruments Corporation, Holtsville, NY) based on phase analysis light scattering via ZetaPALS particle sizing software. Prior to analysis, cuvettes were cleaned with demineralized water and ethanol and stored dry. $1.7 \mathrm{~mL}$ of unfiltered sample solution was placed into clean and dried plastic cuvette. The DLS instrument analyzed each sample for at least three runs at 5 minutes per run. The multiple runs resulted in a combined output reading for the hydrodynamic diameter, $D_{H}$. The DLS 
software also used a dust filter algorithm based on standard deviation to exclude particles of unusually large size.

\subsection{Surface potential measurements}

The surface potentials $(\zeta)$ for PGM, PAH15, PAH900, L-PEI10, B-PEI10, PLL23, PLL230 solutions and their mixtures were determined with a ZetaPALS instrument (Brookhaven Instruments Corporation, Holtsville, NY) using $\zeta$ potential analyzer software. Disposable cuvettes and the electrode were cleaned with Millipore water, ethanol, and then blown-dried with nitrogen. About $1.5 \mathrm{~mL}$ of sample was placed in the cuvette and then the electrode was carefully placed in the cuvette avoiding any spillage. The electrode was always preconditioned in $0.5 \mathrm{M} \mathrm{KCl}$ solution for one cycle consisting of 300 runs before starting any measurement. Ten measurements were taken per sample.

\subsection{Optical waveguide lightmode spectroscopy (OWLS)}

OWLS experiments were carried out using an OWLS 210 Label-free Biosensor system (Microvacuum Ltd, Budapest, Hungary). OWLS is based on grating-assisted in-coupling of a HeNe laser into a planar waveguide coating (200-nm thick Si0.25Ti0.75 $\mathrm{O}_{2}$ waveguiding layer on $1 \mathrm{~mm}$ thick AF 45 glass, Microvacuum Ltd, Budapest, Hungary).

Waveguides used in this study were coated with an ultrathin layer $(\sim 30 \mathrm{~nm}[10])$ of polystyrene (Sigma Aldrich, Brøndby, Denmark) and a subsequent ultrathin layer of PDMS. Polystyrene was prepared at $6 \mathrm{mg} / \mathrm{mL}$ in toluene and spin-coated at $2500 \mathrm{rpm}$ for $15 \mathrm{~s}$. The PDMS base and curing agent (Sylgard 184 elastomer kit, Dow Corning, Midland, MI) were dissolved in hexane at a ratio of 
10:3 (final concentration, $0.5 \% \mathrm{w} / \mathrm{w}$ ), spin-coated onto the waveguide at $2000 \mathrm{rpm}$ for $25 \mathrm{~s}$, and cured in an oven at $70{ }^{\circ} \mathrm{C}$ overnight.

The PS/PDMS-coated waveguide was first exposed to PBS until a stable baseline was obtained. A programmable syringe pump (Model 1000-NE, New Era Pump Systems, Inc., NY) was employed to transport buffer solutions through flow cell containing the OWLS waveguide surface. $100 \mu \mathrm{L}$ of solution sample was then injected via a loading loop. Upon observing an increase in surface adsorption, the pump was stopped for 30 minutes to allow for the adsorption in a static environment. After rinsing the flow cell with PBS, the adsorbed mass density data were calculated according to de Feijter's equations. The refractive index increment $(\mathrm{d} n / \mathrm{d} c)$ values, which are needed for the calculation of the adsorbed masses, were determined for each solution experimentally using an automatic refractometer (Rudolph, J157). The values determined were $0.125,0.147,0.141,0.47$, $0.27,0.223$ and $0.224 \mathrm{~cm}^{3} / \mathrm{g}$ for PGM, PAH15, PAH900, L-PEI10, B-PEI10, PLL23, and PLL230, respectively.

\subsection{Circular dichroism (CD) spectroscopy}

Circular dichroism (CD) spectra were acquired in a quartz cuvette with $1 \mathrm{~mm}$ path length (Hellma GmbH \& Co. KG, Müllheim, Germany) using a Chirascan spectrophotometer (Applied Photophysics Ltd, Surrey, UK). Total polymer concentration in the cuvette was $1 \mathrm{mg} / \mathrm{mL}(\mathrm{w} / \mathrm{v})$ for all samples. Far UV CD spectra were recorded from 280 to $190 \mathrm{~nm}$ with step size of $1 \mathrm{~nm}$ and bandwidth of $1 \mathrm{~nm}$ and near UV CD spectra were recorded from 400 to $250 \mathrm{~nm}$ with step size of 1 $\mathrm{nm}$ and bandwidth of $1 \mathrm{~nm}$. The measurements were performed at room temperature $\left(22{ }^{\circ} \mathrm{C}\right)$. The presented data are average of two independent measurements, each averaged of five scans. 


\section{Results}

\subsection{Friction measurements by pin-on-disc tribometry}

Figure 1 shows the tribological properties of the PDMS/PDMS sliding interface in solutions of PGM or its mixtures with PAH15, PAH900, L-PEI10, B-PEI10, PLL23, and PLL230 at $[\mathrm{PGM}] /[$ Polymer $]=1(\mathrm{w} / \mathrm{w})$ ratio $(1 \mathrm{mg} / \mathrm{mL}$ in total polymer concentration $)$.

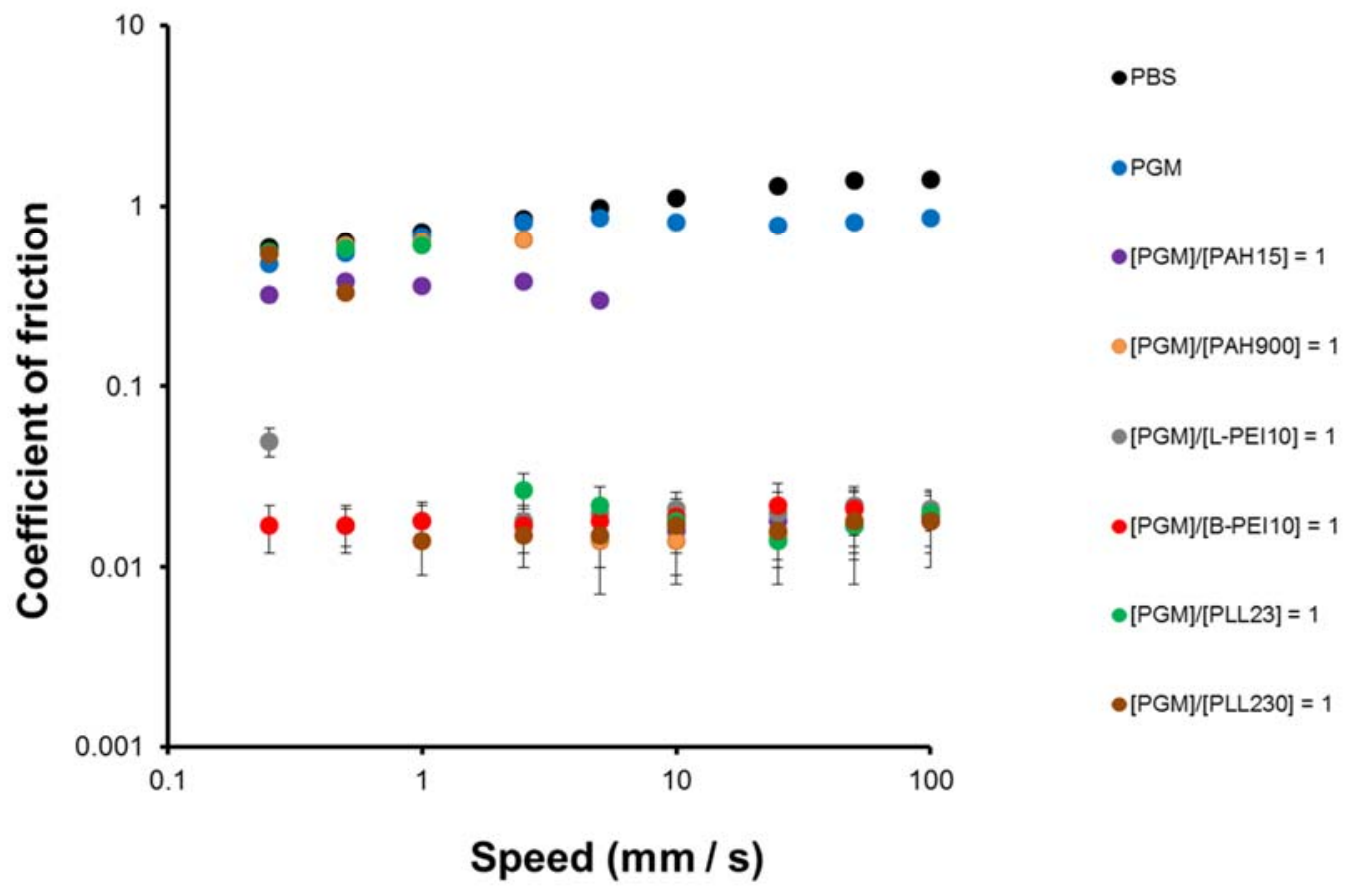

Figure 1. Coefficient of friction vs. speed plots from PDMS/PDMS sliding interface as characterized with pin-on-disc tribometry at $1 \mathrm{~N}$ load for PGM and its mixtures with PAH15, PAH900, L-PEI10, B-PEI10, PLL23, and PLL230 at $[\mathrm{PGM}] /[$ Polymer $]=1$ weight ratio. The error bars for high COF data are typically smaller than each data symbol sizes.

The coefficients of friction obtained for solutions containing only PGM or the polymer (before mixing), namely the two PAHs and two PLLs (Figure 2) as well as two PEIs (not shown), were high and very close to those of buffer solution (PBS) in the entire speed range of the study. The 
poor lubricating properties of PGM are in agreement with past studies at this concentration and $\mathrm{pH}$ [10]. All mixtures showed a decrease in friction of about two orders of magnitude in the high speed regime $(\geq 10 \mathrm{~mm} / \mathrm{s}$ ) where the lubricating capabilities of the various mixtures were indistinguishable from each other. As the sliding speed decreased and the boundary lubrication mode was activated, friction increased to values close to those of buffer in all cases, with the notable exception of the PGM:B-PEI10 mixture, for which the coefficient of friction remains at ca. 0.02 to the lowest speed in this study, i.e. $0.25 \mathrm{~mm} / \mathrm{s}$. The improvement in the boundary lubricating properties of PGM:L-PEI10 was also significant although somewhat inferior to that of PGM:BPEI10. It should be noted that this synergetic lubricating effects of PGM:PEIs, especially PGM:BPEI10, was observed only when PGM and polymers were present in bulk solutions at a sufficiently high concentration $(1 \mathrm{mg} / \mathrm{mL}$ in this study). Exchange of polymer solution with buffer, leaving a monolayer of PGM:PEIs on the PDMS surfaces, did not improve lubricating properties compared to PBS.

One possible reason for the insufficient boundary lubricating properties of most of the PGM:polycation mixtures may be that their optimum mixing ratio for best lubricating efficiency may be not 1:1 w/w ratio that was employed for the experiments in Figure 2. Thus, the mixtures of PGM with PAH15, PAH900, PLL23, and PLL230 were also examined at different weight ratios, yet PGM being still dominant in the overall composition (Figure 2). It is to note that pure polymer solutions were not presented as the polymers alone do not show lubricating properties at PDMS/PDMS interface. As can be seen in Figure 2, the best lubricity is manifested at the $[\mathrm{PGM}] /[$ Polymer $]=1 \mathrm{w} / \mathrm{w}$ ratio for all the polycations studied. 

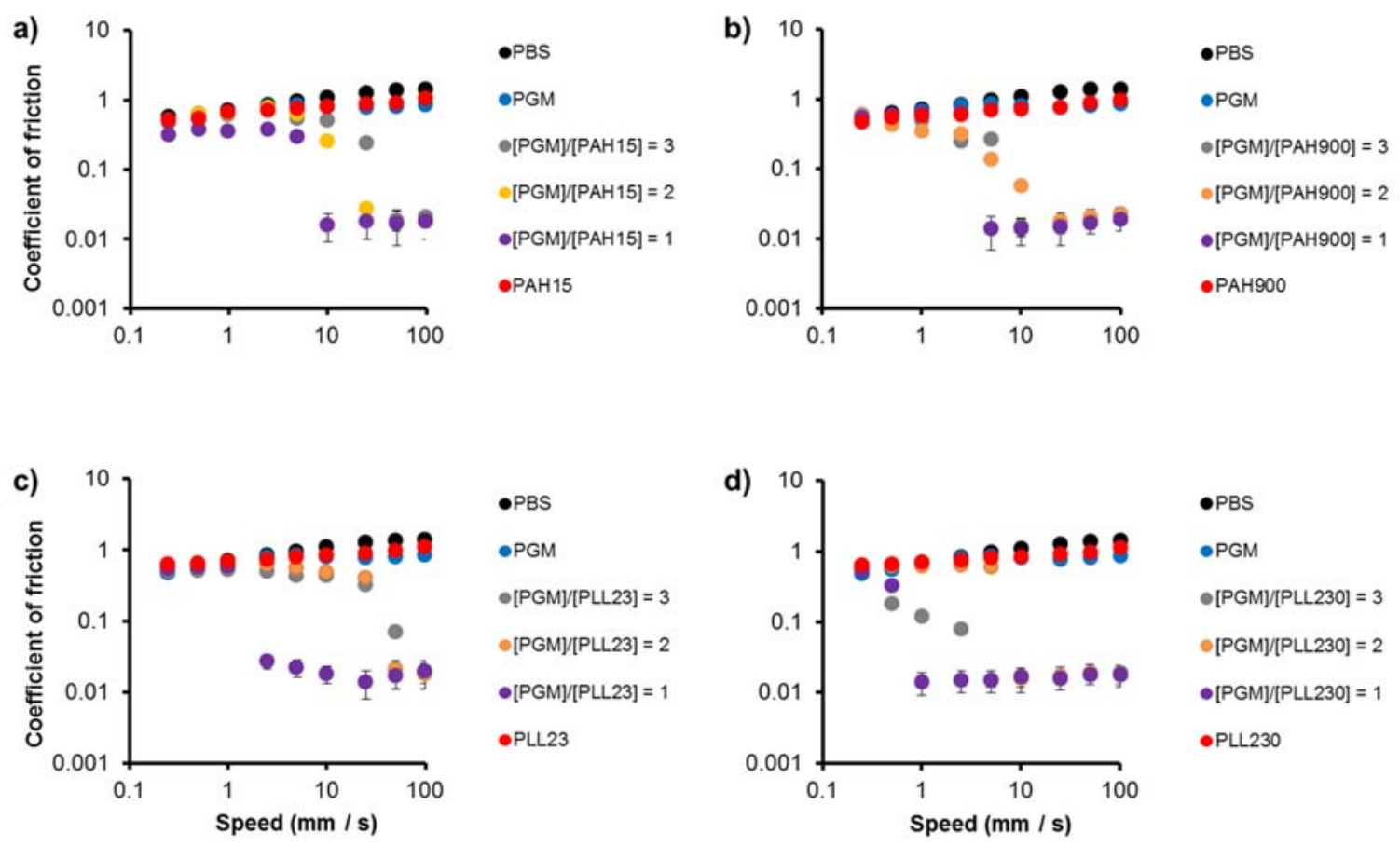

Figure 2. Coefficient of friction vs speed data of PDMS/PDMS sliding interface as characterized with pin-on-disc tribometry at $1 \mathrm{~N}$ load obtained in the polymeric solutions of: a) PGM and PAH15, b) PGM and PAH900, c) PGM and PLL23, d) PGM and PLL230. Mixtures are denoted by the $[\mathrm{PGM}] /[$ Polymer] weight ratio. The error bars for high COF data are typically smaller than each data symbol sizes.

\subsection{Adsorption on PDMS studied by OWLS}

Figure 3 shows the adsorbed masses of PGM, PAH15, PAH900, L-PEI10, B-PEI10, PLL23, PLL230 and the $[\mathrm{PGM}] /[$ Polymer] $=1 \mathrm{w} / \mathrm{w}$ mixtures on PDMS surface as characterized by OWLS. 


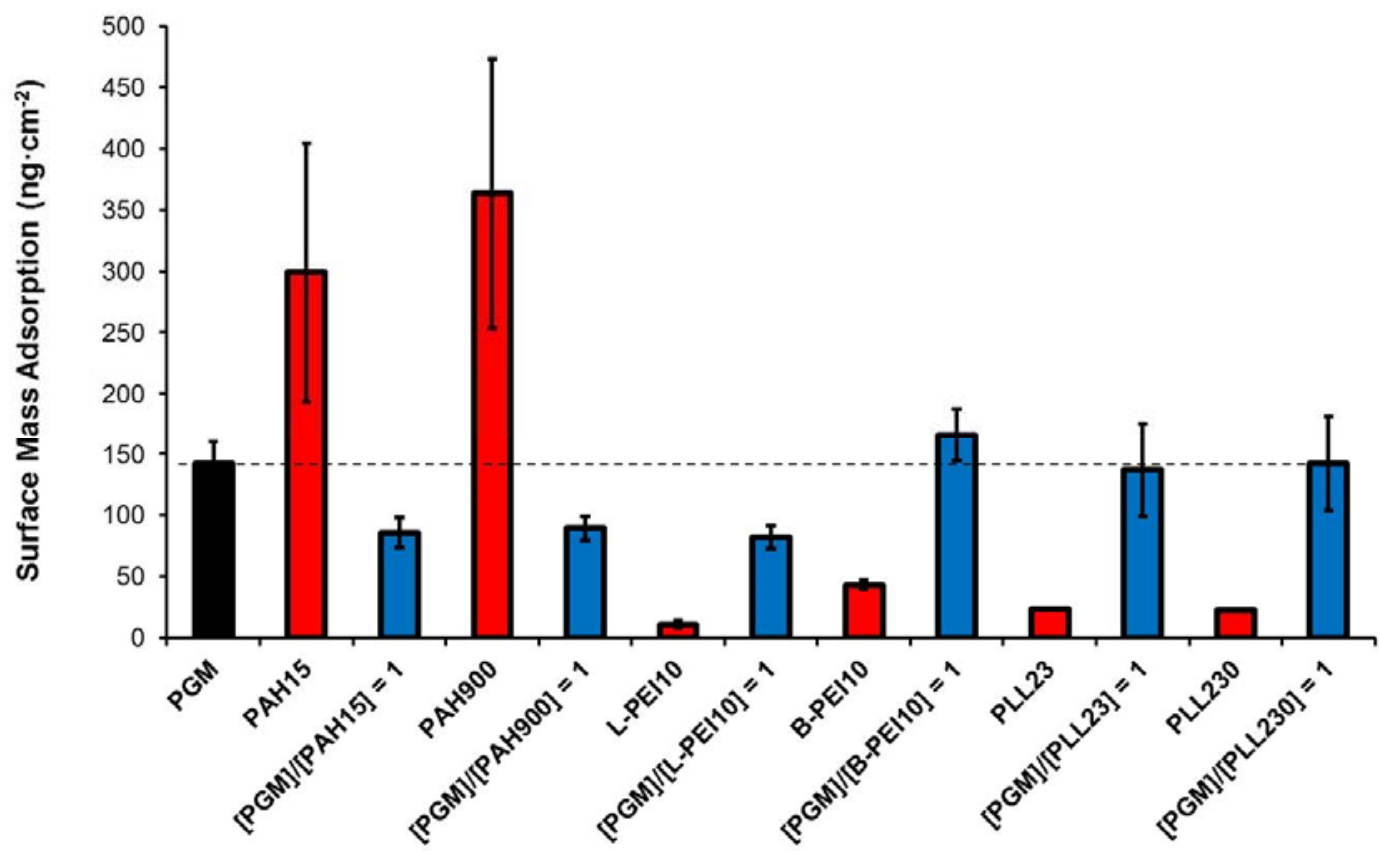

Figure 3. Surface adsorbed masses on PDMS-coated waveguides as characterized by OWLS at pH 7.2 from PGM, PAH15, PAH900, L-PEI10, B-PEI10, PLL23, and PLL230 solutions and the $[\mathrm{PGM}] /[$ Polymer $]=1 \mathrm{w} / \mathrm{w}$ mixtures (concentration $=1 \mathrm{mg} / \mathrm{mL}$ in all cases). A horizontal line (dotted black) indicates the average adsorbed mass of PGM.

The single component polymer solutions can be divided into two groups according to their adsorption behavior onto the PDMS surface. The first group, including L-PEI10, B-PEI10, PLL23, and PLL230, shows very low adsorbed masses, namely less than $30 \mathrm{ng} / \mathrm{cm}^{2}$, whereas the second group, including PGM, PAH15 and PAH900, shows the adsorbed masses higher than ca. 150 $\mathrm{ng} / \mathrm{cm}^{2}$. The low adsorbed masses for the first group are understandable in terms of the strong hydrophilicity of the polymers which leads to weak interaction with the PDMS, as well as the positive charge characteristics which increases the repulsion between the adsorbed molecules onto the nonpolar PDMS surface. Meanwhile, the unglycosylated hydrophobic patches in the C- and Ntermini of PGM function as anchoring units onto the PDMS surface and lead to higher adsorbed masses [1,23-25]. PAH15 and PAH900 do not possess distinct localized hydrophobic patches as 
PGM, yet showed the highest adsorbed mass onto the PDMS $\left(\geq 300 \mathrm{ng} / \mathrm{cm}^{2}\right)$. This can be attributed to the hydrophobic backbone chain of $\mathrm{PAH}$, allowing for the hydrophobic interaction with the PDMS surfaces in aqueous environment [26]. However, due to the possible complexation of PAH with phosphate ions in PBS discussed in detail below, the adsorption mechanism of PAH onto PDMS surface may be quite complex.”

Interestingly, the influence of mixing with PGM on the adsorbed mass changes was opposite for these two groups. For L-PEI10, B-PEI10, PLL23, and PLL230, the adsorbed mass increased significantly as a result of interaction with PGM, whereas for PAH15 and PAH900 rather decreased substantially. The adsorbed masses of the mixtures of PGM with the former group ranged from ca. 70 to $150 \mathrm{ng} / \mathrm{cm}^{2}$, which represent three- to four-fold increase, and those for the latter were slightly less than $100 \mathrm{ng} / \mathrm{cm}^{2}$, which represents three- to four-fold decrease. In view of PGM, however, the changes in the adsorbed mass as a result of mixing with polymers were not as drastic. An exception was the PGM:B-PEI10 mixture which led to an increase of the adsorbed mass by $17 \%$. The mixing of PGM with PAH15, PAH900, or L-PEI10 led to a decrease in the adsorbed mass by ca. $35 \%$ on average, and the mixing of PGM with PLL23 or PLL230 showed comparable adsorbed mass to PGM alone. It is thus important to note that changes in adsorbed mass were not directly correlated with improvement of lubricating properties (Figs 1-2).

\subsection{Hydrodynamic radius and surface potential}

Figure 4 shows hydrodynamic diameter $\left(D_{H}\right)$ distribution of PGM, PAH15, PAH900, L-PEI10, BPEI10, PLL23, PLL230, and their mixtures with PGM at 1 [PGM]/[Polymer] weight ratio. 

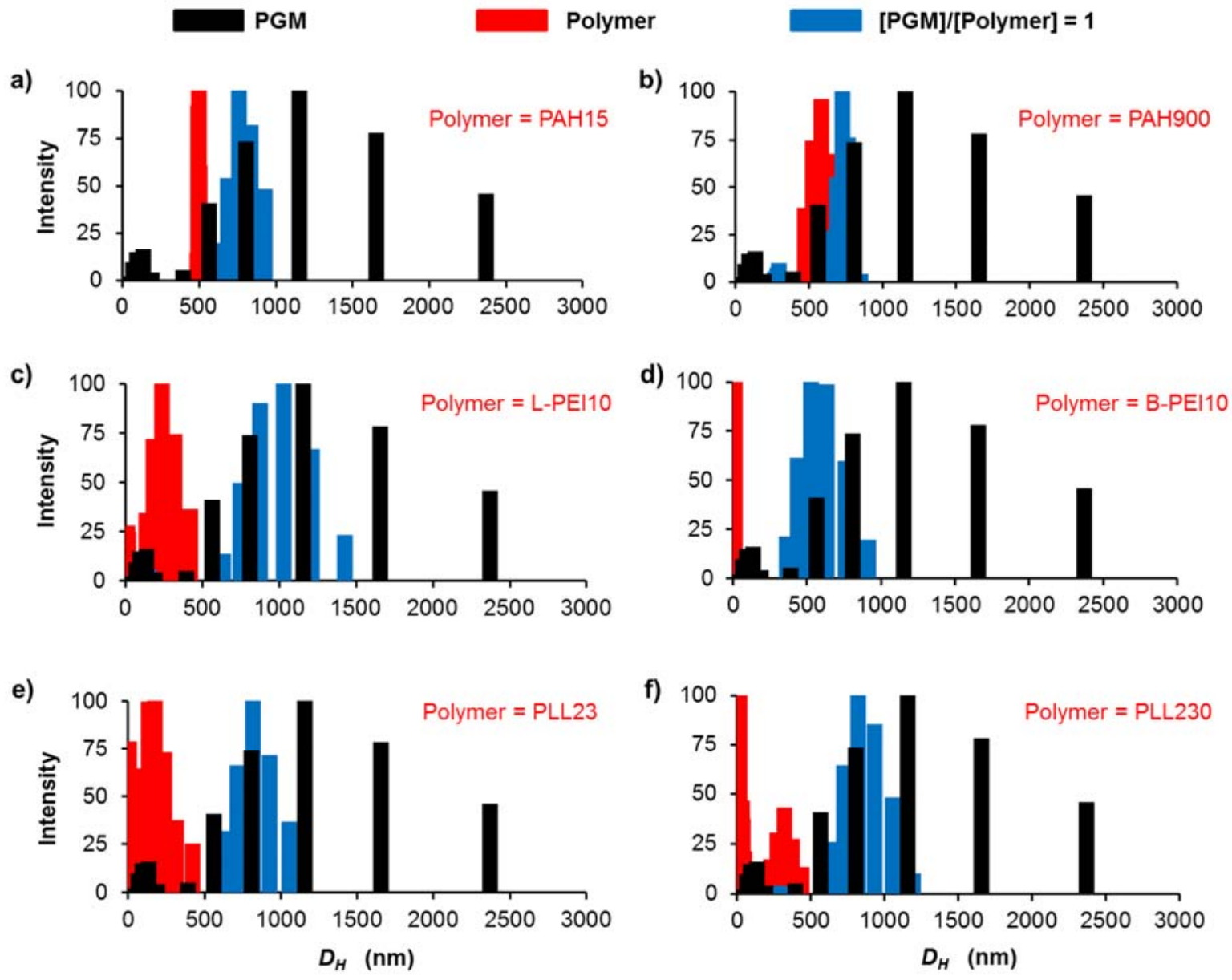

Figure 4. Intensity-weighted distribution of the hydrodynamic diameter $D_{H}$ for PGM and mixtures with (a) PAH15, (b) PAH900, (c) L-PEI10, (d) B-PEI10, (e) PLL23, and (f) PLL230 at $[\mathrm{PGM}] /[$ Polymer] $=1 \mathrm{w} / \mathrm{w}$, as characterized by DLS.

The distribution of $D_{H}$ of PGM molecules is quite broad, reflecting its polydispersity in size. The Zaverage $D_{H}$ values for the samples examined by DLS are listed in Table 1, also showing the $\zeta$ potential of the samples. 
Table 1. $D_{H}$ and $\zeta$-potential averaged values for PGM, PAH15, PAH900, L-PEI10, B-PEI10, PLL23, PLL230, and their mixtures at 1 [PGM]/[Polymer] weight ratio.

\begin{tabular}{|c|c|c|}
\hline Samples & $D_{H}(\mathrm{~nm})$ & $\zeta$-potential $(\mathrm{mV})$ \\
\hline PGM & $1439 \pm 735$ & $-11.0 \pm 1.0$ \\
\hline PAH15 & $495 \pm 11$ & $40.9 \pm 1.7$ \\
\hline$[$ PGM]/PAH15] $=1$ & $793 \pm 115$ & $-3.1 \pm 1.6$ \\
\hline PAH900 & $588 \pm 100$ & $43.5 \pm 1.5$ \\
\hline$[$ PGM]/PAH900] =1 & $737 \pm 57$ & $-6.2 \pm 1.7$ \\
\hline L-PEI10 & $262 \pm 97$ & $11.7 \pm 1.1$ \\
\hline$[$ PGM]/[L-PEI10] $=1$ & $1014 \pm 244$ & $-0.5 \pm 1.4$ \\
\hline B-PEI10 & $13 \pm 2$ & $8.9 \pm 1.1$ \\
\hline$[$ PGM]/[B-PEI10] $=1$ & $605 \pm 168$ & $-2.9 \pm 1.4$ \\
\hline PLL23 & $178 \pm 66$ & $12.6 \pm 1.0$ \\
\hline$[$ PGM]/[PLL23] $=1$ & $840 \pm 160$ & $-4.2 \pm 1.6$ \\
\hline PLL230 & $323 \pm 76$ & $13.8 \pm 3.0$ \\
\hline$[$ PGM]/[PLL230] $=1$ & $869 \pm 168$ & $5.3 \pm 1.5$ \\
\hline
\end{tabular}

All single component polymeric solutions exhibit a diameter lower than that of PGM, with B-PEI10 being the lowest $(13 \pm 2 \mathrm{~nm})$. It is to note that PAH15 are exceptionally monodispersed with the $D_{H}$ of $495 \pm 11 \mathrm{~nm}$, whereas the $D_{H}$ of all the other polymers was more broadly distributed. More importantly, the measured Z-average of $D_{H}$ is too high to be regarded as the hydrodynamic size of single polymer chains of PAH15, considering its relatively small molecular weight [27]. It should also be stressed that the turbidity of PAH solutions mentioned above (section 2.1) was observed only when the polymers were dissolved in PBS. In neat water, e.g. Millipore water, however, PAH solutions were transparent and revealed much smaller $D_{H}$ distributions (Figure S1, Supplementary Data). These observations suggest that phosphate ions in PBS may form complexes with multiple PAH molecules via electrostatic attraction. It has been well known that PAH can form complex structures not only with polyanions with comparable size $[28,29]$, but also with much smaller anionic surfactants, such as potassium hydrogen phthalate [30] or sodium dodecyl sulfate [31]. In 
fact, the complexation with phosphate ions can occur with all the other polycations even though the magnitude/pattern of complexation and its portion in the polymer solution could be significantly different for each case. This effect is most outstanding in the case of PAH15 for its relatively smaller molecular weight and the prevalence of the complex with nearly no free PAH15 unimers in PBS. Even though the detailed structure of PAH:phosphate complex was not determined, the protonated amines groups are expected to be residing on the surface, judging from very highly positive $\zeta$-potentials to be discussed below (Table 1). In all cases, upon mixing PGM with the polymers, the distribution of $D_{H}$ of PGM shifted towards lower values, while the $D_{H}$ peaks of the single polymeric solution vanished. As observed in the previous study of PGM interacting with chitosan [22], the result of the interaction of PGM with all the polycations in this study is the contraction in its size, indicating a strong attraction. This is expected since all polymers in this study are positively charged while PGM is negatively charged at $\mathrm{pH}$ 7.2. Thus, when the positively charged molecules interact with PGM, they can form aggregates via electrostatic attraction, leading to shrinkage in $D_{H}$ of PGM. For the case of PAHs, it remains unclear if PAH:phosphate complex structure is sustained or disassembled to interact with PGM in the course of forming aggregates based on DLS data alone. Lastly, PGM:B-PEI10 is the aggregate with the smallest hydrodynamic diameter, less than half of that of PGM alone.

As expected, all polycations exhibited positive surface charges, while PGM showed a negative one. In all the mixtures, surface charge compensation was observed, e.g. shift from -11.0 mV for PGM towards the less negative values for PGM:polymer mixtures. Even a charge inversion was observed for PGM:PLL230. The changes in $\zeta$-potentials are not expected to provide stand-alone information to assess the interaction characteristics of oppositely charged particles in a mixture. However, given the contraction in $D_{H}$ upon mixing PGM and the polymers (Figure 4), the changes in $\zeta$-potentials of the PGM:polymer solutions may be considered as a result of aggregation between them. For 
instance, the PGM:L-PEI10 aggregates show the surface potential closest to zero, with PGM:BPEI10 and PGM:PAH15 being the next closest ones to surface electroneutrality. Nevertheless, the trends in surface potential of the aggregates do not relate directly to those of the individual components. For example, although PAH900 has the highest positive surface potential as single component in this study, the aggregate that shows the highest positive potential after mixing with PGM is PGM:PLL230 rather than PGM:PAH900. This indicates that not only the net surface charges, but also structural features may play an important role in determining the surface charges of the resulting PGM:polycation aggregates.

\subsection{Conformational changes: CD spectroscopy}

CD spectroscopy is a powerful tool to characterize the conformational features of proteins [32]. A previous study has shown that $\mathrm{CD}$ spectroscopy can be further exploited to probe the conformational changes upon interaction of proteins with polymeric substances [33]. We have thus carried out CD spectroscopic measurements on PGM, PAH15, PAH900, B-PEI10, L-PEI10, PLL23, PLL230, and the mixtures of PGM:Polymers at $1 \mathrm{w} / \mathrm{w}$ weight ratio, to investigate the impact of mixing on the conformation of PGM. The far UV CD spectra (Figure 5) reflect the secondary structure of proteins such as PGM and PLL. 
a)
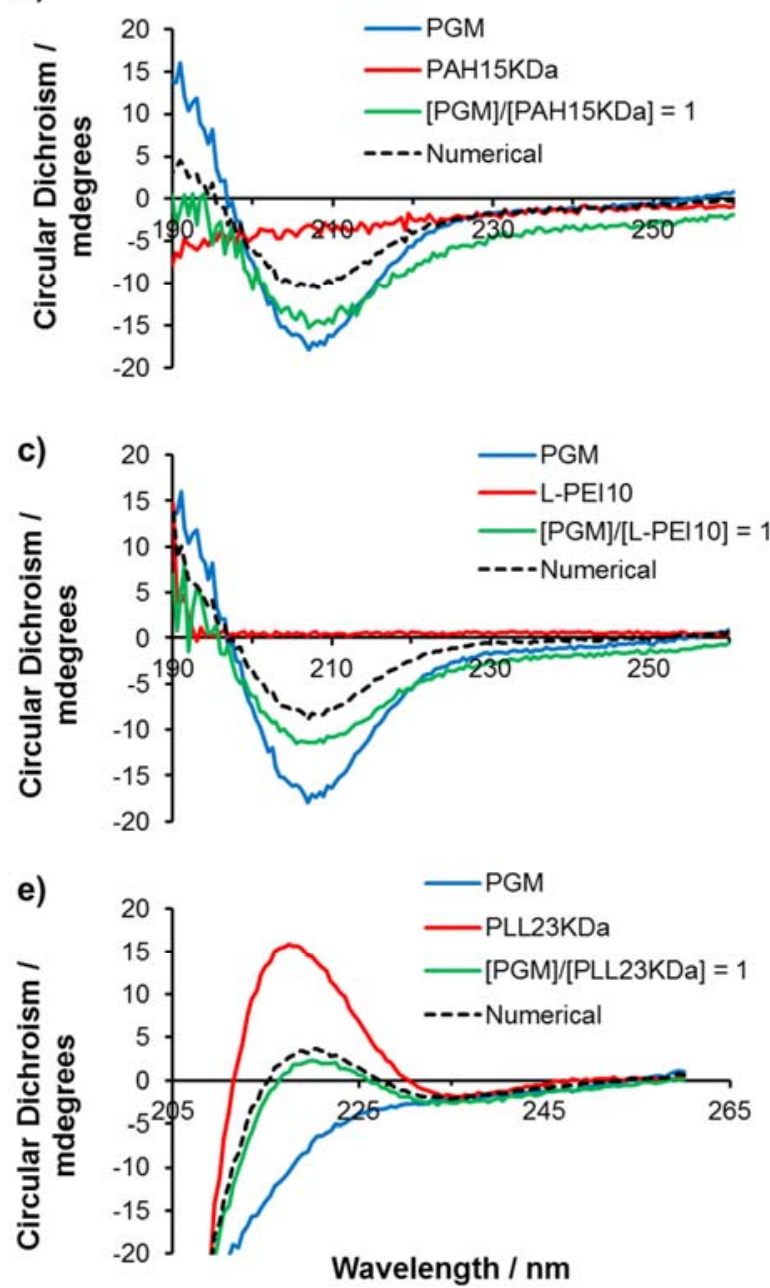

b)

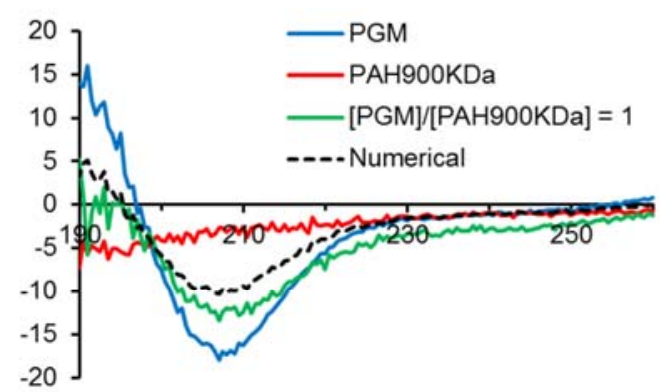

d)

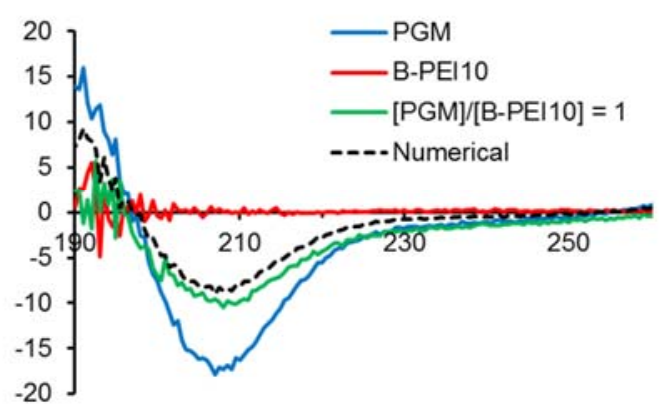

f)

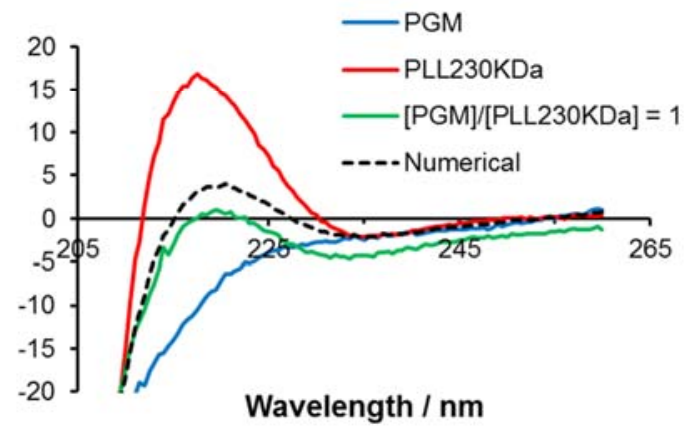

Figure 5. Far UV CD spectra of PGM and the mixtures with (a) PAH15, (b) PAH900, (c) B-PEI10, (d) L-PEI10, (e) PLL23, and (f) PLL230 at [PGM]/[Polymer] = $1 \mathrm{w} / \mathrm{w}$. Blue line: signal of PGM, SPGM, red line: signal of polymer, SPOL, green line: signal of PGM:Polymer mixture as obtained experimentally, SEXP, dashed black line: Numerical value for PGM:Polymer mixture calculated as $\mathrm{S}_{\mathrm{NUM}}=0.5 \cdot\left(\mathrm{S}_{\mathrm{PGM}}+\mathrm{S}_{\mathrm{POL}}\right)$. Exceptionally for the graphs with PLL23 and PLL230, the horizontal axes start at $200 \mathrm{~nm}$ due to very high noise in the spectra at lower wavelengths.

PGM showed a negative peak centered at $206 \mathrm{~nm}$, characteristic of an overall random coil peptide chromophores [10]. Both PLL samples displayed a prominent positive peak with the maximum at around $220 \mathrm{~nm}$, which was assigned to "extended helix" structure in previous studies [34,35]. While 
a strong negative peak is also reported with minimum at ca. $195 \mathrm{~nm}[34,35]$, the relatively high concentration in this sample appeared to scatter the CD signals in the lower wavenumber region $(<$ ca. $205 \mathrm{~nm}$ ), and thus, they were not presented in the spectra, Figure 6 (e-f). Meanwhile, all synthetic polymers, including PAHs and PEIs, showed negligible or completely absent far UV CD signals. The concentration of chromophores in the mixtures of PGM:synthetic polymer in this study, i.e. from PGM, is half that of the neat PGM solution $(1 \mathrm{mg} / \mathrm{mL})$. Therefore, PGM in these mixtures should display half the signal of neat PGM. If SPGM and Spol are denoted as the signal of neat PGM solution (blue line in Figure 5) and that of the polymer (red line in Figure 5) at $1 \mathrm{mg} / \mathrm{mL}$, respectively, then the numerical average of them $\left(\mathrm{S}_{\mathrm{NUM}}\right)$ is calculated as $\mathrm{S}_{\mathrm{NUM}}=0.5 \cdot\left(\mathrm{S}_{\mathrm{PGM}}+\mathrm{S}_{\mathrm{POL}}\right)$ (black, dotted line in Figure 5). If no interaction between them takes place, $\mathrm{S}_{\mathrm{NUM}}$ should be equal to that of the experimentally determined CD spectra of PGM:polymer mixtures ( $\mathrm{S}_{\mathrm{EXP}}$, green line in Figure 5) as the contribution of the polymer in the mixture to CD spectra would be absent other than in halving of the PGM signals due to dilution. As shown in Figure 6 (a)-(d) though, the experimentally acquired far-UV CD spectra for all the PGM:synthetic polymers are distinguished from the numerical averages of the two. In general, the absolute intensities of SEXP are larger than those of $\mathrm{S}_{\mathrm{NUM}}$ although the magnitude of difference varied depending on the polymer mixed with PGM. Higher intensities of the negative peaks centered at ca. $206 \mathrm{~nm}$ indicate that the structure of PGM:polymer aggregates was more compact than that of neat PGM [36]. Thus, it can be concluded that the randomly coiled PGM structure was contracted as a result of mixing with the four types of synthetic polymers in this study. For the cases of the two PLLs (Figure 5(e) and (f)), not only PGM, but also PLL displays a characteristic peak in the region from ca. $210 \mathrm{~nm}$ to $230 \mathrm{~nm}$. Nevertheless, the difference between $\mathrm{S}_{\mathrm{NUM}}$ and $\mathrm{S}_{\mathrm{EXP}}$ for the mixtures PGM:PLL23 and PGM:PLL230 was as clear as for the other mixture solutions, indicating that conformational changes due to interaction with the proteins also occurred in these cases. 

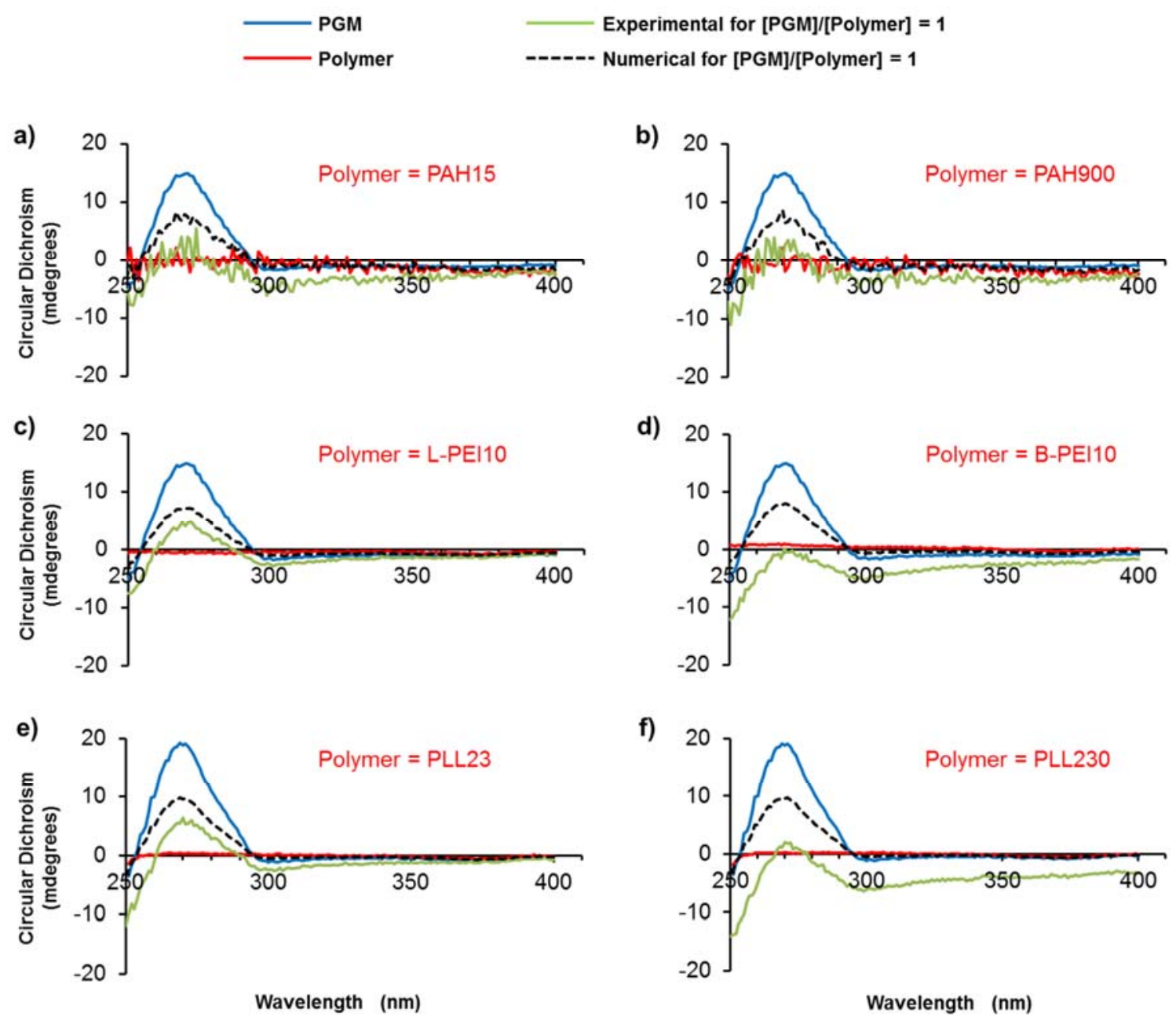

Wavelength $(\mathrm{nm})$

Figure 6. Near UV CD spectra of PGM and the mixtures with (a) PAH15, (b) PAH900, (c) LPEI10, (d) B-PEI10, (e) PLL23, and (f) PLL230 at [PGM]/[Polymer $]=1 \mathrm{w} / \mathrm{w}$. Blue line: signal of PGM, SPGM, red line: signal of polymer, SPOL, green line: signal of PGM:Polymer mixture as obtained experimentally, SEXP, dashed black line: Numerical value for PGM:Polymer mixture calculated as $\mathrm{S}_{\mathrm{NUM}}=0.5 \cdot(\mathrm{SPGM}+\mathrm{SPOL})$.

Figure 6 shows the near UV spectra of PGM, PAH15, PAH900, L-PEI10, B-PEI10, PLL23, PLL230, and their mixtures of $[\mathrm{PGM}] /[$ Polymer $]=1$ weight ratio. Near UV spectra are known to reflect the tertiary structure of proteinaceous macromolecules [37]. In particular, near UV CD 
signals in 260 to $280 \mathrm{~nm}$ are generally assigned to the aromatic side chains [32], which are present only in the $\mathrm{C}$ - and N-terminal regions of mucins. Thus, the prominent positive peak at around 270 $\mathrm{nm}$ in near UV CD spectrum of PGM can be attributed to tertiary structural features caused by the hydrophobic moieties in the terminal regions. Moreover, the appearance of near UV CD signals exclusively from PGM in this study allows for an easy tracking of its conformational changes upon interaction with the polycations. In our study, SEXP deviated from the $\mathrm{S}_{\mathrm{NUM}}$ for all mixtures, and generally $\mathrm{S}_{\text {EXP }}$ had smaller peak intensities than $\mathrm{S}_{\mathrm{NUM}}$. This indicates that in all cases the interaction of PGM with cationic polymers results in weakening the tertiary structure of PGM in the terminal regions. Diminished charge characteristics of PGM as a result of interaction with polycations drive reduced tendency to shield hydrophobic moieties at the terminal regions, and thus exposed aromatic side chains to solvent can freely move to weaken chirality and peak intensities at around $270 \mathrm{~nm}$. In other words, polycations act similarly with acids in weakening the tertiary structure at the terminal regions of PGM [10]. This deviation is largest for the case of PGM:B-PEI10 and PGM:PLL230 aggregates, which means that B-PEI10 and PLL230 are the polymers that have the strongest effect on the tertiary structure in the terminal regions of PGM.

\section{Discussion}

The interaction of PGM with various cationic polymers was examined with an emphasis on the lubricating efficiency of PGM:polymer mixtures at the PDMS/PDMS sliding interface in neutral $\mathrm{pH}$ aqueous solution. Although many of the polycations considered in this study showed a strong interaction with PGM, only mixtures with PEIs and particularly B-PEI10 led to a significant improvement of lubrication in the boundary lubrication. Namely, the coefficient of friction decreased from ca. 0.60 to 0.02 for speeds as low as $0.25 \mathrm{~mm} / \mathrm{s}$ for the case of B-PEI10. 
Given that all polymers are positively charged and PGM is negatively charged under the experimental conditions in this study, electrostatic attraction between PGM and the polycations play a major role in the interaction mechanism between them. This is supported by the contraction of the PGM:polycation due to decreased intramolecular electrostatic repulsion of the PGM molecules and formation of aggregates with the polycations (Figure 4) and positive shift of the overall surface potential ( $\zeta$-potential) due to surface charge compensation (Table 1). Stronger intensities of the negative peak at $208 \mathrm{~nm}$ in the far UV CD spectra of PGM:polymer mixtures, SeXP, than the calculated average of them, SNUM (Figure 5), are consistent with the contracted hydrodynamic size of PGM by DLS, because the absolute signal intensity of the random coil features of mucins is greater when the structure is more compact [36]. However, the fact that the surface charges of the polycations ( $\zeta$-potentials) (Table 1$)$ do not appear to linearly correlate with the resulting effects in surface recharge, hydrodynamic size, and conformation of the PGM:polymer aggregates also suggests that the electrostatic attractions between PGM and the polymer do not suffice to explain the changes addressed above. In fact, the interaction of PGM with these polymers at $\mathrm{pH} 7$ can be enabled via not only electrostatic attraction but also other interaction forces such as hydrogen bonding, Van der Waals forces, and hydrophobic interactions. For example, changes in tertiary structure of PGM after mixing with polymers (Figure 6) suggest that hydrophobic interactions may operate between PGM and the polycations. The near UV CD signal in the region $260-280 \mathrm{~nm}$ is sensitive to the tertiary structural features surrounding aromatic side groups, which are known to be present exclusively in the terminal regions of mucins, although electrostatic attractions can also occur in this region. On the other hand, hydrophobic interactions alone cannot account for the interaction either, since the increase of $\mathrm{Mw}$ of PAH and PLL, both comprising hydrophobic groups, did not reveal any appreciable effects in the interaction with PGM. Moreover, the abundance of 
various hydrogen bonding donors and acceptors in both the amine-based polycations and PGM may readily contribute to the interaction between them.

Effective interaction between PGM and polycations in bulk solution can affect their lubricity mainly because of two reasons. Firstly, it affects the adsorption process onto the PDMS surface, in terms of both amount and stability. Among the polycations examined, the two PAHs are expected to have the highest positive charge density (Table 1), as the degree of ionization of amine groups in $\mathrm{PAH}$ at neutral $\mathrm{pH}$ typically reaches ca. 85\% [26]. In comparison, the degree of ionization of amine groups for B-PEI and L-PEI at neutral $\mathrm{pH}$ is reported to be only ca. $50 \%$ and $30-45 \%$, respectively [26], due to the presence of secondary or tertiary amines, which may also account for their lowest positive $\zeta$-potentials (Table 1 ). However, polycations with higher positive charge density do not necessarily lead to a more favorable interaction with PGM; too highly positive $\zeta$-potential of polycations (e.g. ca. $41 \mathrm{mV}$ for PAH15 and $43 \mathrm{mV}$ for PAH900) compared to the $\zeta$-potentials of PGM, ca. $-11 \mathrm{mV}$ at $\mathrm{pH} 7.2$ (Table 1), may result in weaker interaction due to insufficient charge compensation. In this sense, it should be noted that the $\zeta$-potentials of the aggregates of PGM:LPEI10 and PGM:B-PEI10 are closest to neutral, i.e. cancelling out the charges of the two oppositely charged macromolecules most effectively. This is important for favorable adsorption of PGM, polycations or their aggregates onto the PDMS surfaces as the adsorption of charged macromolecules onto nonpolar surfaces tends to be hindered by electrostatic repulsion between them on the surface $[38,39]$. For example, the increase of the adsorbed masses of PGM:PEIs and PGM:PLLs compared to those of their respective component (Figure 3) can be correlated with the accompanying shift of their $\zeta$-potentials towards zero (Table 1). Ultimately, this is one of the important contributing factors to the improved lubricating properties of PGM:polymer aggregates [39]. A notable exception is the adsorption behavior of PAHs; as shown in Figure 3, the adsorbed masses of PAH15 and PAH900 decrease drastically upon mixing with PGM, even though their $\zeta-$ 
potentials also shifted significantly towards zero (Table 1). The adsorption behavior of PAH and its aggregates with PGM onto PDMS surface is particularly complicated due to the presence of PAH:phosphate complex in PBS. A distinct turbidity of the PAH solutions in PBS indicates that PAH:phosphate complex is overall less soluble due to higher hydrophobicity and it drives a substantially higher amount of adsorption onto PDMS surface. Nevertheless, as the positive charge moieties $\left(-\mathrm{NH}_{3}{ }^{+}\right)$and hydrophobic hydrocarbon backbones are located very close to each other, a high charge density/electrostatic repulsion is also expected on non-polar substrate surfaces, leading to a weak adsorption of PAH. The complete lack of lubricity (Figure 2) despite enormous amount of adsorbed mass (Figure 3) of both PAH molecules alone supports the weak nature of the surface adsorption onto PDMS surface. Thus, the surface adsorption of PGM:PAH mixture can be mainly driven by free or liberated PGM from the aggregates. Furthermore, the hydrodynamic sizes of the two PAHs are the largest of all the polycations examined, which is also resulted from the formation of PAH:phosphate complex involving multiple PAH molecules as mentioned above. Moreover, the shrink in hydrodynamic size upon mixing with PGM is the smallest for PAHs, which further supports that aggregative interaction with PGM is least effective for PAHs or PAH:phosphate complexes. Lastly, this also suggests that the interaction between PAH and PGM, as well as its adsorption and lubricating effects at PDMS-PDMS interface, could be significantly different if aqueous solvent with no or other anions than phosphates are employed.

Secondly, the size and the changes in the size of the polycations upon interaction with PGM are important also because the lubricating layer is not robust enough to withstand the tribostress in the pin-on-disc tribometry experiments. As the pin slides on the PDMS surface, the adsorbed molecules are gradually removed due to tribostress allowing direct PDMS-PDMS contact and the corresponding increase in friction. However, if the aggregates in solution are capable of readsorbing fast enough onto the exposed areas of the PDMS the proteinaceous film is "healed" and 
friction forces can be maintained low $[40,41]$. This is because the adsorption of PGM onto PDMS is initially diffusion-controlled $[23,24]$, and hence, the smaller the aggregates' size, the faster they readsorb onto the surface "healing" the exposed areas. In fact, B-PEI10, which provided the most effective synergy in the lubricating properties upon mixing with PGM, is by an order of magnitude smaller in hydrodynamic diameter $(13 \mathrm{~nm})$ than the rest of the polymers $(178-588 \mathrm{~nm})$ and two orders of magnitude smaller than PGM (1439 nm). The exceptionally smaller hydrodynamic size of B-PEI10, even taking into account the molecular weights of the examined polycations arises from the fact that it is the only branched polymer in this study. While the theoretical degree of branching for B-PEI is $33 \%$ [26], NMR analysis in a previous study on the commercially available branched PEI [42] showed that the degree of branching of B-PEI10 in this study (Sigma) reaches nearly 50\%, which may generate an even denser conformation in bulk solution and reduce the hydrodynamic size. This feature is very advantageous for B-PEI10 to penetrate deeper into the much larger and loose PGM random coil, leading to the enhanced aggregative interaction between them. The smaller size of PGM:B-PEI10 aggregates compared to other aggregates is also an important factor for the synergetic lubricating effects. For larger polycations with sizes comparable to that of PGM, electrostatic attraction between them may occur on the surface level only. For extensive entanglement and/or interpenetration between PGM and such polycations, uncoiling of both macromolecules should accompany in the course of aggregation, which could be thermodynamically rather unfavorable. While the large hydrodynamic sizes of PAHs are chiefly originated from the complexation with phosphate ions, the influence of the polycation size on the efficacy in the interaction with PGM remains valid. The difference of electrostatic interaction between PGM and polycations with significantly different sizes is schematically presented in scheme 2. 


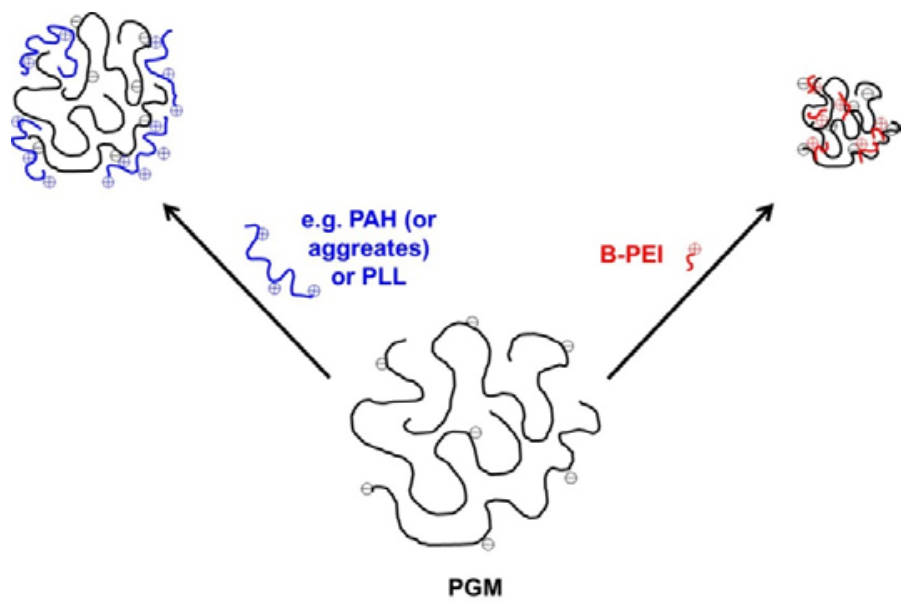

Scheme 2. A schematic illustration of the difference in the interaction modes between PGM and large polycations, such as PAH (or its aggregates) or PLL, vs. small polycation, such as B-PEI, in this study.

\section{Conclusions}

In this study, the lubricating synergy of PGM with a series of cationic polymers, including PAH, PLL, B-PEI and L-PEI, was examined at a hydrophobic interface (PDMS/PDMS). Although all polycations employed in this study interacted with PGM in solution, producing shrinkage of PGM size, surface recharge towards positive values, and distortion of PGM secondary and tertiary structures, B-PEI10 showed an exceptionally superior synergy in lubrication over the entire speed range examined. Namely, in the $[\mathrm{PGM}] /[\mathrm{B}-\mathrm{PEI}]=1 \mathrm{w} / \mathrm{w}$ mixture solution $(1 \mathrm{mg} / \mathrm{mL}$ in total concentration), the friction coefficient was decreased by two orders of magnitude in the speed range of 0.25 to $100 \mathrm{~mm} / \mathrm{s}$ (friction coefficient remained at ca. 0.02). The adsorbed mass of PGM onto PDMS increased only marginally when mixed with the polymers, even for the case of BPEI10, which shows that higher mass adsorption is not a prerequisite of effective lubrication synergy. Most of the PGM:Polymer pairs showed substantial reduction in absolute $\zeta$ potential values upon mixing, but as the PGM:PEI10 pair only showed exceptionally improved lubricating 
properties, charge neutralization upon aggregation is not a sufficient condition for lubrication synergy.

This study shows that the relative size of the PGM and polycations is one of the most crucial parameters for optimum lubricating synergy. When PGM and the polycation are comparable in size, the aggregation between them may occur on the surface level only. However, B-PEI is very compact due to its branched structure as well as the absence of bulky side groups in its monomeric unit, thus, possessing an order of magnitude smallest hydrodynamic diameter than the rest of the polymers. This allows for deeper penetration into the mucinous aggregate, leading to greater collapse of its size and greater distortion of its tertiary structure which results to its superior lubricant synergy. In turn, the most reduced size in PGM:B-PEI10 among all the PGM:polymer aggregates as a result of the most effective aggregation is another crucial factor contributing to effective lubrication synergy. Given that PGM:polymer layers, including PGM:B-PEI10, cannot effectively resist tribostress as monolayer lubricant film in aqueous environment, the ability of the aggregates to reform the lubricant film and "heal" the removed areas due to the cyclic tribostress from the PDMS surface is important. In this process, the smaller the aggregates, the faster they readsorb onto these areas $[22,40,41]$.

While associative interaction between oppositely charged macromolecules can be observed from many pairs, synergetic lubrication as a result of association is relatively much rarer. The present study has shown that there are more requirements for a pair of macromolecules to display synergetic lubricating properties beyond associative aggregation. Comparable charge density in two oppositely charged macromolecules is firstly important, but comparable hydrodynamic size in them can rather act as a barrier for more effective association between them.

\section{Acknowledgement}


The European Research Council (Funding scheme, ERC Starting Grant 2010, Project number 261152) and Novo Nordisk Foundation (Project Number NNF14OC0013309) are acknowledged for their financial support for this work.

\section{References}

[1] R. Bansil, E. Stanley, J.T. LaMont, Mucin Biophysics, Ann. Rev. Physiol. 57 (1995) 635-657.

[2] N.A. Peppas, J.J. Sahlin, Hydrogels as Mucoadhesive and Bioadhesive Materials: A Review, Biomaterials 17 (1996) 1553-1561.

[3] N.A. Peppas, Y. Huang, Nanoscale Technology of Mucoadhesive Interactions, Adv. Drug Deliv. Rev. 56 (2004) 1675-1687.

[4] J.D. Smart, The Basics and Underlying Mechanisms of Mucoadhesion, Adv. Drug Deliv. Rev. 57 (2005) $1556-1568$.

[5] V.V. Khutoryanskiy, Advances in Mucoadhesion and Mucoadhesive Polymers, Macromol. Biosci. 11 (2011) 748-764.

[6] G. Cassin, E. Heinrich, H.A. Spikes, The Influence of Surface Roughness on the Lubrication Properties of Adsorbing and Non-adsorbing Biopolymers, Tribol. Lett. 11 (2001) 95-102.

[7] E. Perez, J.E. Proust, Forces between Mica Surfaces Covered with Adsorbed Mucin across Aqueous Solution, J. Coll. Interf. Sci. 118 (1987) 182-191.

[8] M. Malmsten, E. Blomberg, P. Claesson, I. Carlstedt, I. Ljusegren, Mucin Layers on Hydrophobic Surfaces Studied with Ellipsometry and Surface Force Measurements, J. Coll. Interf. Sci. 151 (1992) 579590.

[9] N.M. Harvey, G.E. Yakubov, J.R. Stokes, J. Klein, Normal and Shear Forces between Surfaces Bearing Porcine Gastric Mucin, a High-Molecular-Weight Glycoprotein, Biomacromolecules 12 (2011) 1041-1050.

[10] S. Lee, M. Müller, K. Rezwan, N.D. Spencer, Porcine Gastric Mucin (PGM) at the Water/ Poly(Dimethylsiloxane) (PDMS) Interface: Influence of $\mathrm{pH}$ and Ionic Strength on Its Conformation, Adsorption, and Aqueous Lubrication Properties, Langmuir 21 (2005) 8344-8353.

[11] I.A. Sogias, A.C. Williams, V.V. Khutoryanskiy, Why is Chitosan Mucoadhesive?, Biomacromolecules 9 (2008) 1837-1842.

[12] B. Menchicchi, J.P. Fuenzalida, K.B. Bobbili, A. Hensel, M.J. Swamy, F.M. Goycoolea, Structure of Chitosan Determines Its Interaction with Mucin, Biomacromolecules 15 (2014) 3550-3558.

[13] S. Rossi, M.C. Bonferoni, G. Lippoli, M. Bertoni, F. Ferrari, C. Caramella, U. Conte, U. Influence of Mucin Type on Polymer-Mucin Rheological Interactions, Biomaterials 16 (1995) 1073-1079. 
[14] M.M. Patel, J.D. Smart, T.G. Nevell, R.J. Ewen, P.J. Eaton, J. Tsibouklis, Mucin/Poly(Acrylic Acid) Interactions: A Spectroscopic Investigation of Mucoadhesion, Biomacromolecules 4 (2003) 1184-1190.

[15] M. Boegh, S.G. Baldursdottir, A. Müllertz, H.M. Nielsen, Property Profiling of Biosimilar Mucus in a Novel Mucus-Containing In-vitro Model for Assessment of Intestinal Drug Absorption, Eur. J. Pharm. Biopharm. 87 (2014) 227-235.

[16] N.D. Catron, H. Lee, P.B. Messersmith, Enhancement of Poly(Ethylene Glycol) Mucoadsorption by Biomimetic End Group Functionalization, Biointerphases 1 (2006) 134-141.

[17] N.V. Efremova, Y. Huang, N.A. Peppas, D.E. Leckband, Direct Measurement of Interactions between Tethered Poly(ethylene glycol) Chains and Adsorbed Mucin Layers, Langmuir 18 (2002) 836-845.

[18] A. Fuongfuchat, A.M. Jamieson, J. Blackwell, T.A. Gerken, Rheological Studies of the Interaction of Mucins with Alginate and Polyacrylate, Carbohyd. Res. 284 (1996) 85-99.

[19] B. Menchicchi, J.P. Fuenzalida, A. Hensel, M.J. Swamy, L. David, C. Rochas, F.M. Goycoolea, Biophysical Analysis of the Molecular Interactions between Polysaccharides and Mucin, Biomacromolecules 16 (2015) 924-935.

[20] P. Sriamornsak, N. Wattanakorn, Rheological Synergy in Aqueous Mixtures of Pectin and Mucin, Carbohyd. Poly. 74 (2008) 474-481.

[21] L. Joergensen, B. Klösgen, A. Cohen Simonsen, J. Borch, E. Hagesaether, New Insights into the Mucoadhesion of Pectins by AFM Roughness Parameters in Combination with SPR, Int. J. Pharm. 411 (2011) 162-168.

[22] N. Nikogeorgos, P. Efler, A.B. Kayitmazer, S. Lee, "Bio-glues" to Enhance Slipperiness of Mucins: Improved Lubricity and Wear Resistance of Porcine Gastric Mucin (PGM) Layers Assisted by Mucoadhesion with Chitosan, Soft Matter 11 (2015) 489-498.

[23] L. Shi, K.D. Caldwell, Mucin Adsorption to Hydrophobic Surfaces, J. Coll. Interf. Sci. 224 (2000) 372381.

[24] L. Lindh, P.-O. Glantz, I. Carlstedt, C. Wickström, T. Arnebrant, Adsorption of MUC5B and the Role of Mucins in Early Salivary Film Formation, Coll. Surf. B: Biointerfaces 25 (2002) 139-146.

[25] A.A. Feiler, A. Sahlholm, T. Sandberg, K.D. Caldwell, Adsorption and Viscoelastic Properties of Fractionated Mucin (BSM) and Bovine Serum Albumin (BSA) Studied with Quartz Crystal Microbalance (QCM-D), J. Coll. Interf. Sci. 315 (2007) 475-481.

[26] S.L. Clark, P.T. Hammond, The Role of Secondary Interactions in Selective Electrostatic Multilayer Deposition, Langmuir 16 (2000) 10206-10214.

[27] B. Jachimska, T. Jasin'ski, P. Warszyn'ski, Z. Adamczyk, Conformations of Poly(Allylamine hydrochloride) in Electrolyte Solutions: Experimental Measurements and Theoretical Modeling, Colloids and Surface A: Physichochem. Eng. Aspects 355 (2010) 7-15. 
[28] R. Chollakup, J.B. Beck, K. Dirnberger, M. Tirrell, C.D. Eisenbach, Polyelectrolyte Molecular Weight and Salt Effects on the Phase Behavior and Coacervation of Aqueous Solutions of Poly(acrylic acid) Sodium Salt and Poly(allylamine) Hydrochloride, Macromolecules 46 (2013) 2376-2390.

[29] J.J. Iturri Ramos, S. Stahl, R.P. Richter, S.E. Moya, Water Content and Buildup of Poly(diallyldimethylammonium chloride)/Poly(sodium 4-styrenesulfonate) and Poly(allylamine hydrochloride)/Poly(sodium 4-styrenesulfonate) Polyelectrolyte Multilayers Studied by an in Situ Combination of a Quartz Crystal Microbalance with Dissipation Monitoring and Spectroscopic Ellipsometry, Macromolecules 43 (2010) 9063-9070.

[30] J. Park, Y.-W. Choi, K. Kim, H. Chung, D. Sohn, Aggregation Processes of a Weak Polyelectrolyte, Poly(Allylamine) Hydrochloride, Bull. Korean Chem. Soc. 29 (2008) 104-110.

[31] E. Yoshida, Self-assembly of Poly(Allylamine Hydrochloride) through Electrostatic Interaction with Sodium Dodecyl Sulfate, Colloid. Polym. Sci. 288 (2010) 1321-1325.

[32] S.M. Kelly, N.C. Price, The Use of Circular Dichroism in the Investigation of Protein Structure and Function. Curr. Protein Pept. Sci. 1 (2000) 349-384.

[33] M. Müller, T. Reihs, W. Ouyang, Needlelike and Spherical Polyelectrolyte Complex Nanoparticles of Poly(1-lysine) and Copolymers of Maleic Acid, Langmuir 21 (2005) 465-469.

[34] M.L. Tifany, S. Krimm, Effect of Temperature on the Circular Dichroism Spectra of Polypeptides in the Extended State, Biopolymers 11 (1972) 2309-2316.

[35] R.A. Gelman, W.B. Rippon, J. Blackwell, Interactions between Chondroitin-6-sulfate and Poly-LLysine in Aqueous Solution: Circular Dichroism Studies, Biopolymers 12 (1973) 541-558.

[36] J.B. Madsen, K.I. Pakkanen, S. Lee, Thermostability of Bovine Submaxillary Mucin (BSM) in Bulk Solution and at a Sliding Interface, J. Coll. Interf. Sci. 424 (2014) 113-119.

[37] B. Ranjbar, P. Gill, Circular Dichroism Techniques: Biomolecular and Nanostructural Analyses - A Review, Chem. Biol. Drug Design 74 (2009) 101-120.

[38] J.G. Göbel, N.A.M. Besseling, M.A. Cohen Stuart, C. Poncet, Adsorption of Hydrophobically Modified Polyacrylic Acid on a Hydrophobic Surface: Hysteresis Caused by an Electrostatic Adsorption Barrier, J. Coll. Interf. Sci. 209 (1999) 129-135.

[39] T. Røn, I. Javakhishvili, K. Jankova, S. Hvilsted, S. Lee, Adsorption and Aqueous Lubricating Properties of Charged and Neutral Amphiphilic Diblock Copolymers at a Compliant, Hydrophobic Interface, Langmuir 29 (2013) 7782-7792.

[40] S. Lee, M. Müller, R. Heeb, S. Zürcher, S. Tosatti, M. Heinrich, F. Amstad, S. Permann, N.D. Spencer, Self-Healing Behavior of a Polyelectrolyte-based Lubricant Additive for Aqueous Lubrication of Oxide Materials, Tribol. Lett. 24 (2006) 217-223. 
[41] N.N. Nikogeorgos, J.B. Madsen, S. Lee, Influence of Impurities and Contact Scale on the Lubricating Properties of Bovine Submaxillary Mucin (BSM) Films on a Hydrophobic Surface, Coll. Surf. B: Biointerfaces 122 (2014) 760-766.

[42] A. von Harpe, H. Petersen, Y. Li, T. Kissel, Characterization of Commercially Available and Synthesized Polyethylenimines for Gene Delivery, J. Contr. Release 69 (2000) 309-322. 